\section{Túnel aorto-ventricular izquierdo e insuficiencia aórtica. Informe de un caso y revisión de la literatura}

\section{RESUMEN}

El túnel aorto-ventricular izquierdo es una patología congénita que conecta la aorta ascendente por encima de la unión sinotubular a la cavidad del ventrículo izquierdo. Su incidencia es $0.001 \%$ de las cardiopatías congénitas. Existen reportes de aproximadamente 130 casos en la literatura especializada mundial. Aunque su causa es desconocida una de las hipótesis es que es el resultado de una incorporación defectuosa del bulbus cordis. La sospecha diagnóstica se basa en la exploración física y el estudio ecocardiográfico. El tratamiento de elección es quirúrgico mediante cierre del túnel aorto-ventricular. Se informa el caso de un niño de 11 años de edad con túnel aorto-ventricular izquierdo, con insuficiencia aórtica severa tratada con cierre en el extremo aórtico del túnel y prótesis valvular aórtica.

Palabras clave: túnel aorto-ventricular izquierdo, insuficiencia aórtica, cierre con doble parche.
Jesús Eduardo Méndez-Santos ${ }^{1}$ Héctor Osnaya-Martínez ${ }^{2}$ Alfredo Bobadilla-Aguirre ${ }^{3}$

${ }^{1}$ Médico residente de Cardiología Pediátrica.

2 Médico Adscrito del Servicio de Cardiología Pediátrica.

${ }^{3}$ Jefe del Servicio de Cardiología Pediátrica. Instituto Nacional de Pediatría.

\section{Left aorto-ventricular tunnel with aortic insufficiency. Case report and literature review}

\begin{abstract}
The left aorto-ventricular tunnel is a congenital condition that connects the ascending aorta above the sinotubular junction to the left ventricular cavity. Its incidence is $0.001 \%$ of all congenital heart disease. There are only about 130 cases reported in the literature. Although its etiology is unknown, one hypothesis is the result of a defective incorporation of bulbus cordis. The diagnosis is suspected based on physical examination and echocardiography findings. The treatment of choice is surgical trough closure of aorto-ventricular tunnel. We report the case of an 11 year old male with left aorto-ventricular tunnel with severe aortic regurgitation treated with tunnel closure at the aortic end aortic valve prosthetic replacement.
\end{abstract}

Key words: Left aorto-ventricular tunnel, Aortic regurgitation, Double patch closure.
Recibido: 1 de octubre del 2013

Aceptado: 20 de junio del 2014

Correspondencia: Dr. Jesús Eduardo Méndez Santos

Insurgentes Sur 3700-C

CP 04530 México, D.F.

Tel: 1084-0900, Ext: 1228

jesus_sants@hotmail.com

Este artículo debe citarse como

Méndez-Santos JE, Osnaya-Martínez H, BobadillaAguirre A. Túnel aorto-ventricular izquierdo e insuficiencia aórtica. Informe de un caso y revisión de la literatura. Acta Pediatr Mex 2015;36:26-31. 


\section{INTRODUCCIÓN}

El túnel aorto-ventricular izquierdo es una rara malformación cardiaca congénita que consiste en una comunicación entre la aorta ascendente, por encima de la unión sinotubular, y los ventrículos izquierdo o derecho. Tiene una incidencia en pacientes con cardiopatía congénita de $0.001 \%$; se han reportado tan sólo 130 casos en la literatura especializada mundial; es dos veces más frecuente en hombres que en mujeres.

Levy y sus colaboradores, en 1963, informaron una comunicación paravalvular entre la aorta ascendente por encima de las arterias coronarias, sin pasar por la válvula aórtica, que terminaba en el ventrículo izquierdo generando una fisiología que imitaba una insuficiencia aórtica. ${ }^{1}$ Embriológicamente se han propuesto varias teorías al desarrollo de esta patología, entre ellas resaltan una incorporación defectuosa en el extremo distal del bulbus cordis. ${ }^{2}$ Los autores de este primer estudio sugirieron que el túnel era la representación de una arteria coronaria anómala y que su dilatación posterior era un fenómeno adquirido. ${ }^{1}$ Por otra parte, McKay informó que el sustrato para su formación, junto con sus lesiones asociadas, puede inferirse en el desarrollo anatómico, los cojines endocárdicos que forman el aparato valvular aórtico y pulmonar con sus respectivas valvas se separan por un plano de tejido extracardiaco debido a la regresión de músculo circundante. La falta de este plano de tejido podría resultar en un túnel por encima de uno de los senos de Valsalva. ${ }^{3}$ En casi $80 \%$ de los casos el túnel aorto-ventricular se origina por encima de la coronariana derecha, que cursa entre el plano de tejido entre la aorta y el infundíbulo pulmonar, para incorporarse al ventrículo izquierdo; casi $90 \%$ de los túneles terminan en el ventrículo izquierdo.

Basado en la morfología, Hovaguimian y sus colegas propusieron una clasificación anatómica:
Tipo 1. En forma de hendidura, apertura en el extremo aórtico sin deformación valvular.

Tipo 2. Un gran aneurisma extracardiaco.

Tipo 3. Aneurisma intracardiaco de la porción septal del túnel, con o sin obstrucción en el tracto de salida del ventrículo derecho.

Tipo 4. La combinación de tipos 2 y 3.

Es probable que las variaciones en la morfología sean manifestaciones del origen y curso del túnel. ${ }^{4}$

Los datos clínicos suelen evidenciar un soplo sistolodiastólico en foco aórtico, con frémito e irradiación a hueco supraesternal, acompañado de pulsos amplios. En pacientes mayores los síntomas pueden ser similares a una estenosis valvular aórtica; sin embargo, se asemejan más a los de una insuficiencia valvular aórtica con alteraciones en la presión arterial sistémica. La gravedad de los síntomas es variable y los pacientes pueden permanecer asintomáticos por años, hasta desarrollar insuficiencia cardiaca con descompensación súbita y muerte. Los estudios de gabinete iniciales son electrocardiograma y radiografía de tórax en proyección posteroanterior, que muestran datos de hipertrofia ventricular izquierda, pueden indicar datos de sobrecarga diastólica y cardiomegalia con dilatación de aorta ascendente, respectivamente. La ecocardiografía transtorácica es la prueba diagnóstica de elección: en eje largo paraesternal y eje corto demuestran el túnel con dilatación de la aorta ascendente. En Doppler color se observa flujo diastólico originado a nivel de la unión sinotubular alejándose hacia el ventrículo izquierdo. Los túneles que se abren hacia el ventrículo derecho se visualizan en el eje corto paraesternal de la válvula aórtica, mientras que la función del ventrículo izquierdo, que es afectada de forma variable con hipertrofia y dilatación, se evalúa en 
la vista paraesternal eje corto a nivel de los músculos papilares. ${ }^{3}$ En casos no concluyentes, en los que no se puedan demostrar con claridad las arterias coronarias, se puede requerir angiografía con catéter. Con estudios avanzados como la resonancia magnética también se pueden demostrar los túneles aorto-ventriculares derecho o izquierdo. El diagnóstico diferencial incluye defecto septal ventricular con lesión valvular, doble lesión aórtica, aneurisma o rotura del seno de Valsalva.

Para el tratamiento de esta cardiopatía se recomienda el cierre quirúrgico en el momento del diagnóstico, independientemente de la edad y los síntomas, debido a la poca efectividad de tratamiento médico para estabilizar los síntomas y el riesgo de desarrollar insuficiencia aórtica severa en pacientes atendidos tardíamente. ${ }^{5}$ El objetivo de cualquier tratamiento es obliterar el túnel. Las técnicas que se han descrito son cierre del orificio de la aorta con o sin un parche, cierre del extremo ventricular del túnel, obliteración del túnel (ligadura del túnel o resección parcial del túnel) y cierre con doble parche de ambos orificios (aórtico y ventricular). ${ }^{6}$ Otra opción de tratamiento que puede ser aplicable a estos pacientes es el cierre transcutáneo con oclusor Amplatzer ${ }^{\circledR}$ PDA en pacientes seleccionados, en ausencia de otras afecciones asociadas. ${ }^{7}$

El daño de la válvula aórtica es frecuente dada la lesión paravalvular. Es tratada con medidas que van desde comisurotomía, reconstrucción de la válvula compleja, aortoventriculoplastia y reemplazo valvular. Otras de las lesiones infrecuentes es la dilatación aneurismática del túnel aorto-ventricular. ${ }^{8}$

En la actualidad la supervivencia, después de la reparación quirúrgica, ha aumentado $20 \%$ a casi $100 \%$. Una pequeña cantidad de pacientes, en asociación con displasia de la válvula aórtica con origen aberrante de las coronarias o el origen de una sola arteria coronaria, presentan alto riesgo quirúrgico ${ }^{9}$ mientras que la mortalidad es pequeña con insuficiencia aórtica significativa. ${ }^{4}$

\section{MATERIAL Y MÉTODOS}

Se revisaron todos los pacientes sometidos a reemplazo valvular aórtico en el Instituto Nacional de Pediatría entre los años 1993 y 2013. Se reportaron 8 casos con reemplazo valvular y en uno se realizó el diagnóstico de túnel aorto-ventricular. Se hizo una descripción del caso y revisión del tema.

\section{Descripción del caso}

Niño de 11 años de edad referido de un hospital de segundo nivel con diagnóstico de estenosis valvular aórtica. En sus antecedentes se refiere disnea de esfuerzo en clase funcional II de la New York Heart Association de 2 años de evolución. En la exploración física presentó una frecuencia cardiaca de 90 latidos por minuto, tensión arterial 108/44, frémito en hueco supraesternal, precordio hiperdinámico, soplo sistolodiastólico en foco aórtico y accesorio aórtico IV/VI que irradia a hueco supraesternal, signos de Corrigan, Musset, Quincke y Traube presentes.

Los estudios de gabinete mostraron en la radiografía de tórax cardiomegalia y crecimiento de los perfiles derecho e izquierdo (Figura 1). El electrocardiograma mostró hipertrofia ventricular izquierda y sobrecarga de volumen en precordiales izquierdas. El ecocardiograma transtorácico en eje largo paraesternal mostró un flujo que se originaba a nivel de la coronariana derecha y se presenta durante la diástole con dirección hacia el ventrículo izquierdo, anillo valvular aórtico dilatado con protrusión hacia el ventrículo izquierdo (Figura 2A). En el ecocardiograma en eje corto paraesternal en modo bidimensional y Doppler color se observó el anillo aórtico dilatado con flujo diastólico entre coronarias derecha e izquierda (Figura 2B). En una vista supraesternal de eje largo se observó 


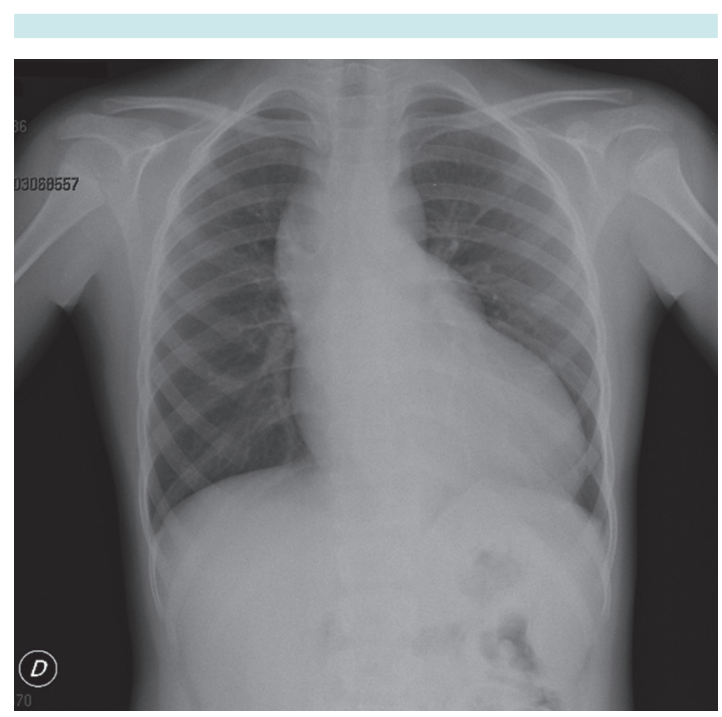

Figura 1. Radiografía de tórax en proyección posteroanterior con índice cardiotorácico 0.65; imagen sugestiva de dilatación de aorta ascendente en perfil derecho y botón aórtico dilatado.

una inversión holodiastólica del flujo en la aorta descendente, una pendiente de desaceleración mayor de $3.5 \mathrm{~m} / \mathrm{s}^{2}$ en un eje apical 5 cámaras, y un diámetro diastólico y sistólico con Z score +6.1 que confirmaron dilatación ventricular por insuficiencia aórtica. Por este método no fue posible definir la anatomía de las arterias coronarias y, ante duda de túnel aorto-ventricular izquierdo versus aneurisma roto de seno de Valsalva, se decidió realizar cateterismo cardiaco que mostró patrón coronario normal y en un aortograma en incidencia oblicua anterior derecha donde se observó una estructura vascular externa originada en la raíz de la aorta, que correspondía a un túnel aorto-ventricular. La presión telediastólica del VI fue de $21 \mathrm{mmHg}$ (Figura 3). La resonancia magnética apoyó el diagnóstico de túnel aorto-ventricular izquierdo al observarse una comunicación a nivel de la unión sinotubular hacia el ventrículo izquierdo durante la fase diastólica (Figura 4). Se inició tratamiento anticongestivo y se llevó a cirugía donde se encontró una válvula aórtica bivalva, engrosada y con prolapso, túnel

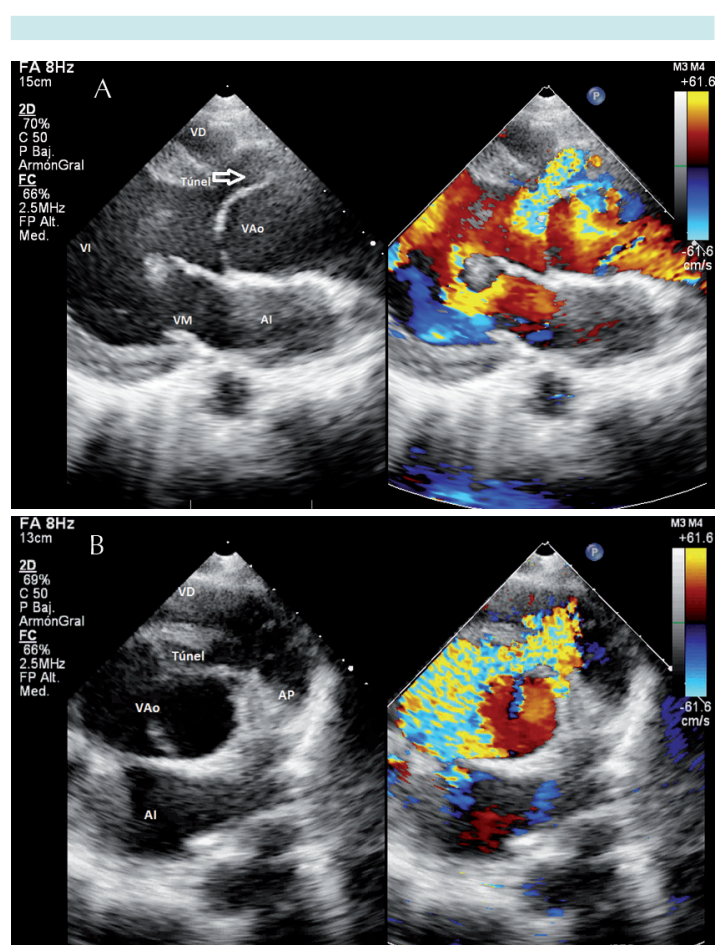

Figura 2. A) Ecocardiograma en modo bidimensional y Doppler color en eje largo paraesternal: túnel aortoventricular con el cortocircuito hacia el ventrículo izquierdo (flecha) y prolapso de la válvula aórtica hacia el ventrículo izquierdo. VD: ventrículo derecho; VI: ventrículo izquierdo; VAo: válvula aórtica; VM: válvula mitral; Al: aurícula izquierda. B) Eje corto paraesternal con cortocircuito entre coronarianas derecha e izquierda. VD: ventrículo derecho; VAo: válvula aórtica; AP: arteria pulmonar; Al: aurícula izquierda.

aorto-ventricular entre la comisura de las valvas coronarianas derecha y la izquierda, ligeramente por debajo de la unión sinotubular. Se colocó parche tratado con glutaldehido en el extremo aórtico del túnel y una prótesis valvular aórtica tipo St. Jude $25 \mathrm{~mm}$. El paciente permanecía asintomático a los 22 meses de la cirugía.

\section{Análisis}

El túnel aorto-ventricular es una cardiopatía congénita poco frecuente descrita por primera vez 


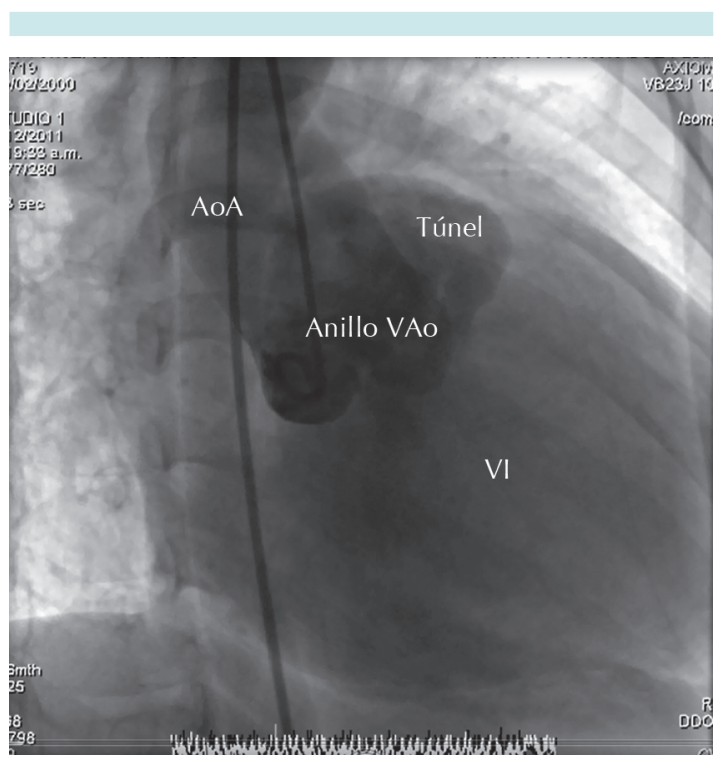

Figura 3. Aortograma en proyección oblicua anterior derecha: túnel aorto-ventricular izquierdo. AoA: aorta ascendente; VAo: válvula aórtica; VI: ventrículo izquierdo.

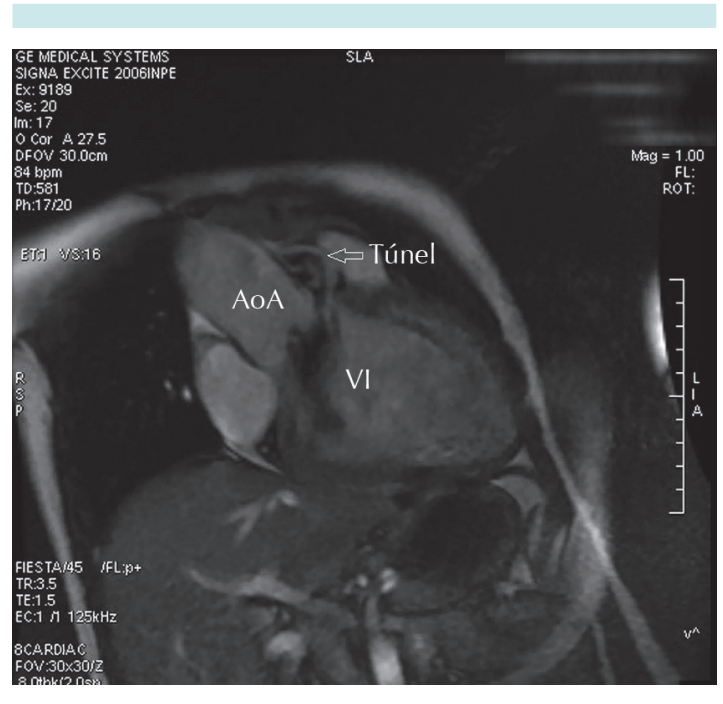

Figura 4. Imagen de resonancia magnética en 3 cámaras: estructura posterior a la aorta con comunicación hacia el ventrículo izquierdo. AoA: aorta ascendente; VI: ventrículo izquierdo. por Levy y sus colaboradores, en 1963, en tres pacientes con estudio patológico. ${ }^{1}$ El estudio con mayor información es el realizado por Martins en el que se identificaron 11 pacientes, entre 1963 y agosto del 2002, con un predominio del sexo masculino; 8 desarrollaron insuficiencia cardiaca congestiva con edades menores de 6 meses y el resto estuvieron clínicamente asintomáticos con edades de 3 años. Todos los pacientes presentaban datos de regurgitación aórtica leve. Entre las lesiones asociadas se encontraron defecto septal interventricular, estenosis aortica y subaórtica critica y fistula coronaria derecha al túnel aorto-ventricular. La técnica quirúrgica realizada en 9 pacientes fue cierre del extremo aórtico del túnel y uno se cerró en el extremo ventricular. Solo un paciente requirió valvuloplastia aórtica con balón. ${ }^{5}$

El síntoma principal en esta afección es la aparición de soplo cardiaco con frémito; sin embargo, se ha visto que puede iniciar con insuficiencia cardiaca congestiva de forma súbita. El estudio diagnóstico de elección es el ecocardiograma transtorácico, en modo Doppler color, que muestra un flujo diastólico a nivel del tracto de salida de ventrículo izquierdo, dirigiéndose hacia el ventrículo izquierdo junto con alteraciones en el anillo aórtico y la raíz aórtica.

El ecocardiograma inicial de nuestro paciente mostró una gran dilatación del anillo aórtico y de la raíz aórtica con un flujo diastólico originado a nivel de la valva coronariana derecha $y$, ante la duda de compromiso con las arterias coronarias, fue llevado a cateterismo cardiaco.

La técnica quirúrgica empleada fue cierre con parche del extremo aórtico del túnel y prótesis valvular aórtica. Si bien existen varias técnicas descritas las más utilizadas son el cierre con doble parche o un solo parche en el extremo aórtico. Otro tratamiento que ha surgido como opción es el cierre con Amplatzer ${ }^{\circledR}$ por técnica 
transcutánea. ${ }^{7}$ Las lesiones asociadas pueden tratarse por separado o en el momento de la reparación del túnel aorto-ventricular.

El túnel aorto-ventricular izquierdo es una patología infrecuente que representa un reto para el pediatra y para el cardiólogo pediatra. El diagnóstico oportuno es importante para evitar complicaciones como la disfunción ventricular y el daño valvular. Se debe sospechar en todo paciente con soplo sistolodiastólico en el que se observe un flujo paravalvular diastólico hacia el ventrículo izquierdo en el estudio ecocardiográfico. El tratamiento definitivo es quirúrgico con corrección de las lesiones asociadas. El pronóstico es favorable a mediano y largo plazos cuando el tratamiento es oportuno.

\section{REFERENCIAS}

1. Levy MJ, Lillehei CW, Anderson RC, Amplatz K, Edwards JE. Aortico-left ventricular tunnel. Circulation 1963;27:841-53.
2. Somerville J, English T, Ross DN. Aorto-left ventricular tunnel Clinical. Features and surgical management. Br Heart J 1974;36:321-8.

3. McKay R. Aorto-ventricular tunnel. Orphanet J Rare Dis 2007;2:41.

4. Mueller C, Dave H, Pretre R. Surgical repair of aortoventricular tunnel. Multimed Man Of Cardiothorac Surg 2012;1-8.

5. Martins JD, Sherwood MC, Mayer JE, Keane JF. Aortico-left ventricular tunnel: 35-year experience. J Am Coll Cardiol 2004;44:446-50.

6. Mitropoulos FA, Laks H, Kanakis MA, Levi D. Aorto-left ventricular tunnel: an alternative surgical approach. Ann Thoracic Surg 2006;82:1113-5.

7. Chessa M, Chaudhari M, De Giovanni JV. Aorto-Left ventricular tunnel: transcatheter closure using an Amplatzer duct occluder device. Am J Cardiol 2000;86:2534.

8. Amoozgar H, Shakibazad N, Amirghofran AA. Aneurysmal aorto-left ventricular tunnel. Int Cardiovasc Res J 2012;6:30-2.

9. Colak N, Nazli Y, Alpay MF, Cakir O. Aorto-left ventricular tunnel with its origin in the left sinus of Valsalva associated with a single coronary artery and aortic insufficiency in an adult. J Card Surg 2011;26:437-9. 